RAD Conference Proceedings, vol. 5, pp. 136-140, 2021

ISSN 2466-4626 (online) | DOI: 10.21175/RadProc.2021.26

www.rad-proceedings.org

\title{
HIGH-FREQUENCY ULTRASOUND SCANNING IN EYELIDS ASSESSMENT
}

\author{
T.N. Kiseleva ${ }^{1}$, Yu. A. Stepanova ${ }^{2}$, N.V. Guseva1, K.V. Lugovkina1, V. V. Makukhina1 \\ ${ }^{1}$ Helmholtz National Medical Research Center of Eye Diseases, Moscow, Russia \\ ${ }^{2}$ A.V. Vishnevsky National Medical Research Center of Surgery, Moscow, Russia
}

\begin{abstract}
The results of high-frequency grayscale B-scan, color Doppler imaging (CDI) and ultrasonic density measurement or echodensitometry (ED) of eyelids and periorbital tissues were presented. 48 healthy volunteers (96 eyes) aged from 17 to 46 years were enrolled. Echographic anatomy of eyelid layers, i.e. skin, orbicularis oculi muscle, tarsus, and Retro-Orbicularis Oculi Fat (ROOF), is described in details. Thickness and echodensitometry values for all layers were provided. Complex ultrasound examination should be performed prior to reconstructive and plastic surgery of eyelids in order to facilitate the choice of surgical tactics.
\end{abstract}

Key words: High-frequency grayscale B-scan, echodensitometry, echographic anatomy of eyelid layers

\section{INTRODUCTION}

It is known that predominantly diagnostics of pathological conditions of eyelids is based on clinical symptoms, ocular examination and the histopathologic findings [1]-[4]. In recent years, there has been an increase in interest in the development of noninvasive and innovative methods of eyelids' examination for the determination of early pathological features.

New ultrasound technologies using high-frequency transducers provide microscopic resolution of images at 40 to 50 micrometer [5]. There are several reports of ultrasound biomicroscopy (UBM) of the eyelid in healthy subjects and in various eyelid disorders [6]-[10]. Kikkawa et al. noticed the correlation between preoperative UBM thickness and histopathological thickness in certain eyelid lesions. They noticed well-defined delineation between malignant and benign lesions in their group of patients [11].

However, there is no literature on normal features of eyelids as assessed by high-resolution grayscale scanning (B-scan) with color Doppler imaging and echodencitometry for identifications of small structures. These techniques are essential and accurate methods in differential diagnosis of neoplastic, tumor-like processes and inflammatory diseases of eyelids.

Diminutiveness and structural characteristics of eyelids provide the complexity in interpretations of ultrasound findings. Moreover in clinical practice the anatomical differentiation of upper and lower eyelids structures is essential for the detection of localization and extension of the pathological process in eyelid disorders. This information will allow clinicians to make a better diagnosis, reduce misdiagnosis and benefit clinical treatment.

\section{PURPOSE}

The purpose is to study the normal ultrasound image of anatomical structures of eyelids using high-frequency ultrasound scanning.

\section{METHODS}

48 healthy volunteers with no eye and eyelid abnormalities aged from 17 to 46 years were observed. After a comprehensive eye examination, an informed consent was obtained and they were subjected to utrasound examinations of the upper and lower eyelids. Ultrasound methods which included high-frequency grayscale B-scan, color Doppler imaging (CDI) and ultrasonic density measurement or echodensitometry (ED) of eyelids and periorbital tissues were performed on the Voluson diagnostic system (GE Healthcare) using linear transducer SP 11-18 MHz with the high resolution zoom function and scanning mode of «Small part». The ultrasound power was reduced according to the ALARA (as low as reasonably achievable) principle. Mechanical index was not more than 0.23 and thermal index was not more than 1.0 [12].

Scans of the eyelids were performed with the volunteer in supine position. The volunteer was instructed to look straightforward and to keep his/her eyes in this direction while they are closed. The ultrasound probe was placed perpendicular to the skin surface using transverse and longitudinal scans. Examination was performed on both sides. Differentiation of eyelids' structures was performed according to its reflectivity (hyperechoic or hypoechoic). The echodensitometry values in conventional units (c.u.) and thickness in millimeters

* tkisseleva@yandex.ru 
for various eyelid structures were recorded. According to the anatomical features the following layers of each eyelid were observed: skin, orbicularis oculi muscle, tarsal plate which peripherally forms the orbital septum. The orbital septum of both superior and inferior eyelids attaches peripherally to the orbital rim bone. Centrally the orbital septum attaches to the junction of the inferior tarsal muscle to the tarsal plate in the lower eyelid and levator palpebrae aponeurosis in the upper eyelid. The thickness of the retroorbicularis oculi fat was measured from the orbicularis oculi muscle to the orbital septum at the level through the superior orbital rim and at the level just below the orbital septum in the lower eyelid, respectively [13]. Differentiation of the septum was more difficult to obtain in the lower lid than in the upper lid.

Color Doppler imaging (CDI) was used for the visualization of vascular structures in eyelid area and measurements of blood flow velocities.

All statistical analysis was carried out using SPSS for Windows version 17.0.

\section{RESULTS}

Considering anatomical data and echographic features of different tissues of eyelids we determined the ultrasound images of eyelid structures and the mean indices of the thickness of eyelid layers in the middle line on longitudinal and transversal scans.

On echograms the first layer, skin, represents a lamellar structure. The surface layer of the skin, epidermis, was hyperechoic and its thickness varied from 0.02 to $0.5 \mathrm{~mm}$. Below, there was the layer corresponding to reticular layer of derma, which showed middle reflectivity and regular structure (Figure 1).

Retro-Orbicularis Oculi Fat (ROOF) appeared to be a hypoechoic regular layer behind orbicularis oculi muscle. The mean thickness of ROOF was

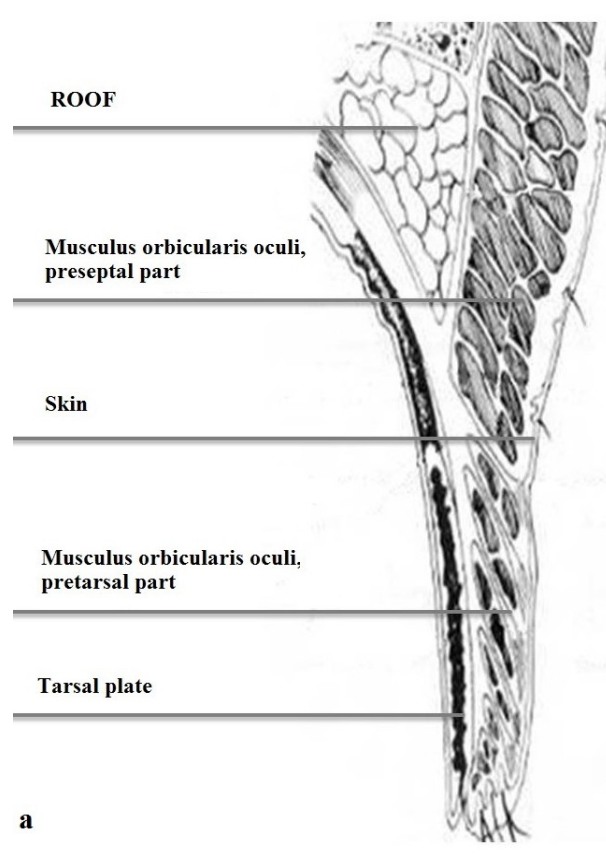

$2.26 \pm 0.4 \mathrm{~mm}$ in upper eyelid and $2.81 \pm 0.6 \mathrm{~mm}$ in lower eyelid. There was no statistically significant difference in the ROOF thickness when compared between upper and lower eyelids.

Our evaluation showed that all eyelid layers could be identified, except for the tarsal muscles, Muller's muscle, levator aponeurosis, capsulopalbebral fascia

CDI and pulse-wave dopplerography demonstrated vessels with arterial blood flow waveform between anterior and posterior lamella of eyelid. These vessels were localized inside fat and divided the eyelid into musculoculaneous and tarso-conjunctival parts.

Measurements of eyelid thickness were performed in the central plane.

The mean thickness of the upper eyelid was $2.90 \pm 0.2 \mathrm{~mm}$, epidermis with derma $-0.98 \pm 0.17 \mathrm{~mm}$, orbicularis oculi muscle $-0.76 \pm 0.1 \mathrm{~mm}$, tarsal plate with conjunctiva $-1.09 \pm 0.15 \mathrm{~mm}$. Results of lower eyelid examination were the following: thickness of eyelid - 3.30 $\pm 0.24 \mathrm{~mm}$, skin with subcutaneous tissue $\quad-\quad 1.12 \pm 0.16 \mathrm{~mm}$, orbicularis oculi muscle $-0.98 \pm 0.14 \mathrm{~mm}$, tarsal plate with conjunctiva $1.19 \pm 0.17 \mathrm{~mm}$. Echodensitometry of eyelid showed follow indices: epidermis $-99.7 \pm 22$ c.u.; papillary dermis layer $-61.6 \pm 18.7$ c.u.; reticular layer of derma

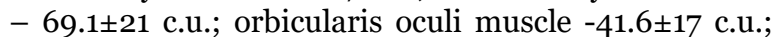
tarsal plate $-97.2 \pm 22$ c.u.

The echographic image of eyelids was lamellar. Epidermis, reticular layer of derma and ROOF, tarsal plate with conjunctiva were hyperechoic. Reticular layer of derma and orbicularis oculi muscle were hypoechoic. There was no statistically significant difference between ultrasonic density indices of the upper eyelid layers and the lower ones. We can use Doppler methods for qualitative and quantitative assessments of eyelids vessels. CDI demonstrated vessels with arterial blood flow waveform between anterior and posterior lamella of eyelid.

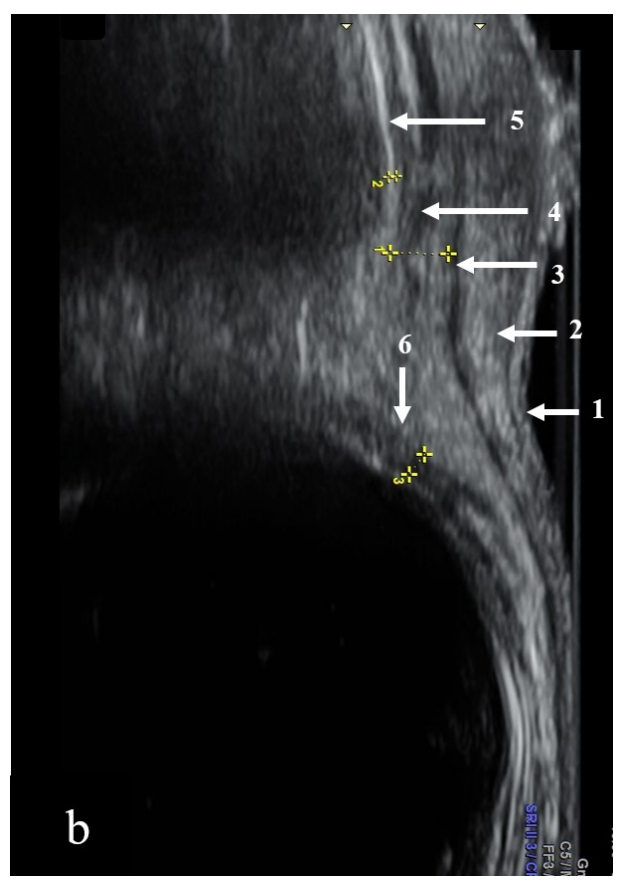


T. Kiseleva et al., High-frequency ultrasound scanning in eyelids assessment, RAD Conf. Proc., vol. 5, 2021, 136-140
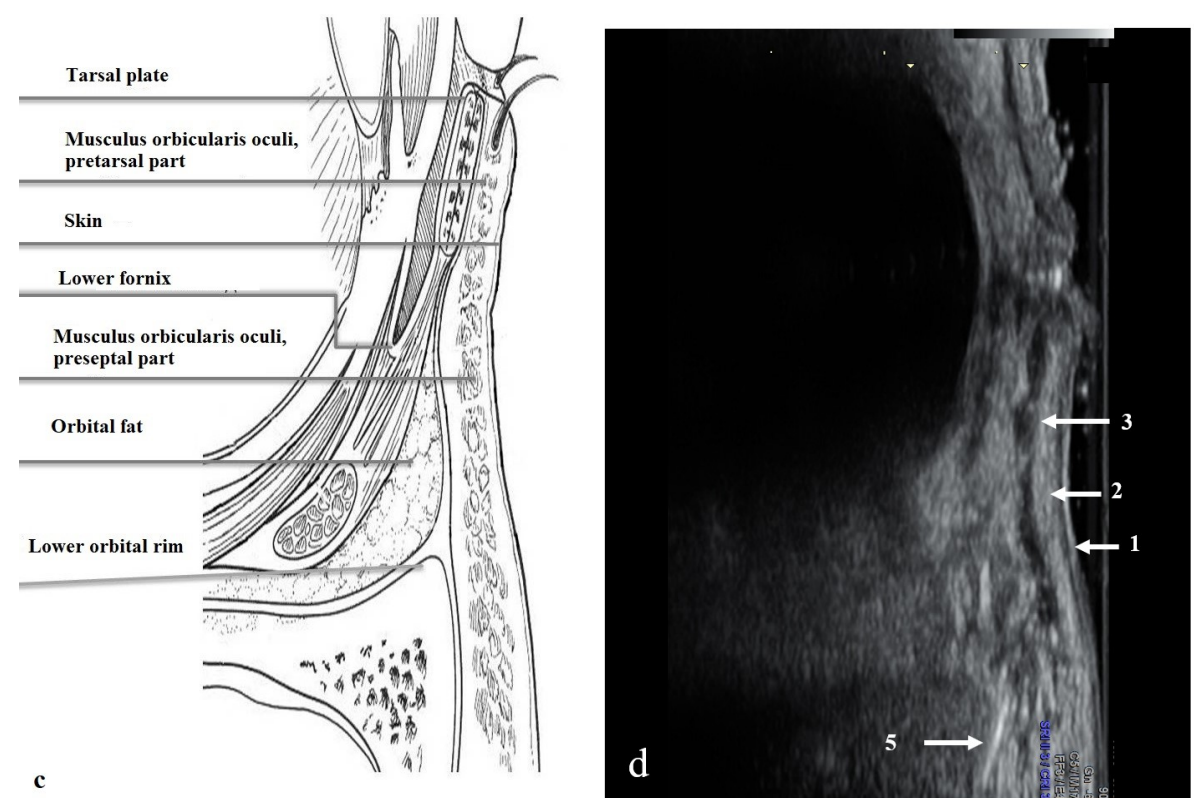

Figure 1. The cartoon diagram represents the area of scan and relevant anatomy for comparison (1a, 1c). Echogram of an upper eyelid (1b). Echogram of lower eyelid (1d).

1 - skin; 2 - subcutaneous tissue; 3 - orbicularis oculi muscle; 4 - ROOF; 5 - septum; 6 - levator palpebrae muscle.

Table 1. Acoustic density of anatomical structures of eyelids

\begin{tabular}{|c|c|c|c|c|c|c|}
\hline \multirow{2}{*}{\multicolumn{3}{|c|}{ Anatomical structures of eyelids }} & \multicolumn{2}{|c|}{ Upper eyelid } & \multicolumn{2}{|c|}{ Lower eyelid } \\
\hline & & & \multirow{2}{*}{$\begin{array}{c}\begin{array}{c}\text { Mean } \\
(\mathrm{M})\end{array} \\
95.4 \pm 20.3\end{array}$} & \multirow{2}{*}{$\begin{array}{c}\begin{array}{c}\text { Standard } \\
\text { error }(\mathrm{m})\end{array} \\
1.6\end{array}$} & \multirow{2}{*}{$\begin{array}{c}\begin{array}{c}\text { Mean } \\
(\mathrm{M})\end{array} \\
94.8 \pm 20.8\end{array}$} & \multirow{2}{*}{$\begin{array}{c}\begin{array}{c}\text { Standard } \\
\text { error }(\mathrm{m})\end{array} \\
1.6\end{array}$} \\
\hline \multirow{6}{*}{$\frac{\Xi}{\mathscr{\sigma}}$} & \multirow{2}{*}{ Epidermis } & Tarsal plate projection & & & & \\
\hline & & $\begin{array}{c}\text { Capsulopalpebral fascia } \\
\text { projection }\end{array}$ & $97 \cdot 5^{ \pm 20.4}$ & 1.5 & $95.4 \pm 22.8$ & 1.7 \\
\hline & \multirow{2}{*}{$\begin{array}{l}\text { Papillary } \\
\text { dermis }\end{array}$} & Tarsal plate projection & $57.4 \pm 18.8$ & 1.2 & $56.7 \pm 16.9$ & 1.2 \\
\hline & & $\begin{array}{c}\text { Capsulopalpebral fascia } \\
\text { projection }\end{array}$ & $59.6 \pm 18.6$ & 1.2 & $57.4 \pm 18.8$ & 1.2 \\
\hline & \multirow{2}{*}{$\begin{array}{l}\text { Reticular } \\
\text { dermis }\end{array}$} & Tarsal plate projection & $81.2 \pm 24.2$ & 1.7 & $82.2 \pm 23.8$ & 1.7 \\
\hline & & $\begin{array}{c}\text { Capsulopalpebral fascia } \\
\text { projection }\end{array}$ & $100 \pm 24.0$ & 1.9 & $104.9 \pm 24.8$ & 2.3 \\
\hline \multirow{2}{*}{ 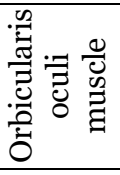 } & \multicolumn{2}{|r|}{ Pre-tarsal part } & $45.2 \pm 14.2$ & 1.1 & $44.8 \pm 14.2$ & 1.1 \\
\hline & \multicolumn{2}{|r|}{ Pre-Septal part } & $46.3^{ \pm 16.8}$ & 1.3 & $46.4 \pm 16.4$ & 1.2 \\
\hline \multicolumn{3}{|c|}{ Tarsal plate } & $98.1 \pm \mathbf{2 2 . 8}$ & 1.7 & $98.2 \pm 23.3$ & 1.7 \\
\hline \multicolumn{3}{|c|}{ ROOF } & $87.5^{ \pm 26.9}$ & 1.9 & $87.2 \pm 27.2$ & 2.0 \\
\hline
\end{tabular}

\section{DisCUSSION}

Eyelid is a complex structure with varied anatomical differences and ultrasonographic properties. This study represents the first report of the echodencitometry, thickness values and Color Doppler assessment of normal eyelid structures as analyzed by high-frequency scanning. We performed the quantitative assessment of ultrasonic density of eyelids structures using two-dimensional histograms accompanied by calculation of mean indices in conventional units (c.u.) of digital image.
Various groups have studied the upper eyelid anatomy as evaluated by UBM $[14,6,7]$.

Demirci et al. studied normal upper eyelid in 16 healthy adult volunteers by randomly choosing one eye. Vasanthapuram V.H. et al presented the first report of echogenecity and measurements of normal lower eyelid using UBM. These authors demonstrated that on UBM grossly three layers were noted: the first hyperechoic layer of skin-orbicularis complex, the second hypoechoic layer of tarsal plate capsulopalpebral fascia, and lastly the third layer hyperechoic comprising conjunctiva and retractor 
(smooth muscle, connective tissue complex). UBM findings can form the basis of normal anatomy for further research on UBM in lower eyelid disorders. Knowledge of the eyelid anatomy and numerous differences between various individuals is vital to achieve the optimal surgical outcome and maintain the ethnical characteristic of an individual. With the recent improvement in technology, UBM could provide a noninvasive, cost-effective alternative to study the eyelid anatomy in different individuals and the changes occurring in various pathologies with enhanced resolution. It has been used to study various eyelid lesions and its correlation with histological characteristics. UBM is a non-invasive imaging tool with good inter-observer correlation.

Unlike the UBM the advantage of high-frequency ultrasound scanning is the visualization of the whole area of eyelid - its height and length including pretarsal and preseptal parts of orbicularis oculi muscle, ROOF and conjunctival fornix.

This method has a few limitations. First, eyelids being a pliable structure, the use of scleral cup or ClearScan cover for UBM may cause intra and interobserver variations in the measurements leading to less repeatability. Second, as UBM was designed for anterior segment imaging, the speed of sound in eyelid tissue is different from the preset value. Third, this method doesn't provide the information about vascular structures.

In our study we used high - frequency ultrasound scanning (11-18 MHz) with the high resolution zoom function. This method was combined with Doppler technology for the visualization of vascular structures and measurement of blood flow velocities. During examination the transducer was applied to the closed eyelids using a thick layer of acoustic gel, with the examiner's hand resting on the orbital margin to minimize the pressure on the eyelids. Our findings were consistent when measured in the central plane. This study showed that various eyelid structures can be definitely identified separately on high-frequency ultrasound imaging with a good correlation of the eyelid anatomy. Thus, this technique allows noninvasive, cost-effective, time-saving alternative for imaging different eyelid structures, studying anatomical variations in individuals and potential use in preoperative assessment in different types of eyelid pathologies. Measurements and descriptions of eyelid structures can form the normative basis for more research on complex high-frequency scanning in eyelid disorders.

\section{CONCLUSION}

Based on the detailed echographic identification of normal anatomic features of eyelid structures, complex ultrasound examination including high frequency B-scan, echodensitometry and CDI can be used successfully in clinical practice. This method is useful in diagnostics of posttraumatic changes, inflammatory lesions, tumors of eyelids.

High frequency ultrasound scanning of eyelids can be recommended prior to reconstructive and plastic surgery in order to facilitate the choice of surgical tactics.

Our study is just a preliminary assessment of eyelid structures using high-frequency ultrasound (11-18 MHz). Further evaluation of its diagnostic precision will be suggested with investigation of a larger number of healthy individuals and patients with different pathologies of eyelids.

\section{REFERENCES}

1. D. Liu, W. M. Hsu, "Oriental eyelids. Anatomic difference and surgical consideration," Ophthal. Plast. Reconstr. Surg., vol. 2, no. 2, pp. 59-64, 1986. http://doi.org/10.1097/00002341-198601050-00001

2. S. Jeong et al., "The Asian upper eyelid: an anatomical study with comparison to the Caucasian eyelid", Arch Ophthalmol., vol. 117, no. 7, pp. 907-912, Jul. 1999. http://doi.org/10.1001/archopht.117.7.907

3. M. Deprez, S. Uffer, "Clinicopathological features of eyelid skin tumors. A retrospective study of 5504 cases and review of literature", Am. J. Dermatopathol., vol. 31, no. 3, pp. 256-262, May 2009. https://doi.org/10.1097/dad.obo13e3181961861

4. L. Wang et al., "Clinicopathological analysis of 5146 eyelid tumours and tumour-like lesions in an eye centre in South China, 2000-2018: a retrospective cohort study," BMJ Open, vol. 11, no. 1, article no. e041854, Jan. 2021 http://doi.org/10.1136/bmjopen-2020-041854

5. A. D. Singh, B. C. Hayden, "Clinical Methods: Ultrasound Biomicroscopy", in Ophthalmic Ultrasonography, Philadelphia, USA: Elsevier/Saunders, 2012, ch. 4, pp. 25-29.

6. V. H. Vasanthapuram, P. Saha, A. Mohamed, M. N. Naik, "Ultrasound biomicroscopic features of the normal lower eyelid," Orbit, vol. 40, no. 5, pp. 375-38o, Sep. 2020. https://doi.org/10.1080/01676830.2020.1812094

7. H. Demirci, C.C. Nelson, "Ultrasound biomicroscopy of the upper eyelid structures in normal eyelids," Ophthal. Plast. Reconstr. Surg., vol. 23, no. 2, pp. 122-125, Apr. 2007. http://doi.org/10.1097/iop.obo13e31802f2074

8. M. T. Rajabi et al., "Ultrasonographic visualization of lower eyelid structures and dynamic motion analysis," Int. J. Ophthalmol., vol. 6, no. 5, pp. 592-595, 2013. http://doi.org/10.3980/j.issn.2222-3959.2013.05.07

9. M. T. Rajabi et al., "Ultrasonographic motion analysis of lower eyelid compartments in patients with chronic thyroid associated ophthalmopathy," J. Curr. Ophthalmol., vol. 29, no. 4, pp. 310-317, Dec. 2017. http://doi.org/10.1016/i.joco.2017.07.002

10. P. Saonanon, P. Thongtong, T. Wongwuticomjon, "Differences between single and double eyelid anatomy in Asians using Ultrasound biomicroscopy," Asia Pac. J. Ophthamol., vol. 5, no. 5, pp. 335-338, Sep-Oct. 2016. http://doi.org/10.1097/APO.0000000000000185

11. D. O. Kikkawa, R. Ochabski, R. N. Weinreb, "Ultrasound biomicroscopy of eyelid lesions," Ophthalmologica, vol. 217, no. 1, pp. 20-23, Feb. 2003. http://doi.org/10.1159/o00068253

12. S.F. Byrne, R.L. Green, "Color Doppler Imaging of the Eye and Orbit", in Ultrasound of the eye and orbit, Philadelphia, USA: Mosby Inc., 2002, ch. 14, pp. 374-375.

13. T.A Ferreira et al., "MR and CT Imaging of the Normal Eyelid and its Application in Eyelid Tumors," Cancers, vol. 12, no. 3, p. 658, Mar. 2020. http://doi.org/10.3390/cancers12030658 
T. Kiseleva et al., High-frequency ultrasound scanning in eyelids assessment, RAD Conf. Proc., vol. 5, 2021, 136-140

14. M. H. Banu, N. G. Ayer, G. Zilelioglu, A. H. Elhan, "Ultrasound biomicroscopy of the levator aponeurosis in congenital and aponeurotic blepharoptosis,"
Ophthalmic Plast. Reconstr. Surg., vol. 20, no. 4, pp. 308-311, Jul. 2004.

https://doi.org/10.1097/01.iop.0000129532.33913.e7 